\title{
Ballistic bunching of photoinjected electron bunches with dielectric-lined waveguides
}

\author{
F. Lemery ${ }^{1}$ and P. Piot ${ }^{1,2}$ \\ ${ }^{1}$ Northern Illinois Center for Accelerator \& Detector Development and Department of Physics, \\ Northern Illinois University, DeKalb, Illinois 60115, USA \\ ${ }^{2}$ Accelerator Physics Center, Fermi National Accelerator Laboratory, Batavia, Illinois 60510, USA
}

(Received 3 July 2014; published 17 November 2014)

\begin{abstract}
We describe a simple technique to passively bunch non-ultra-relativistic $(\lesssim 10 \mathrm{MeV})$ electron bunches produced in conventional photoinjectors. The scheme employs a dielectric-lined waveguide located downstream of the electron source to impress an energy modulation on a picosecond bunch. The energy modulation is then converted into a density modulation via ballistic bunching. The method is shown to support the generation of subpicosecond bunch trains with multi-kA peak currents. The relatively simple technique is expected to find applications in compact, accelerator-based, light sources and advanced beam-driven accelerator methods.
\end{abstract}

DOI: 10.1103/PhysRevSTAB.17.112804

PACS numbers: 29.27.-a, 41.85.-p, 41.75.Fr

\section{INTRODUCTION}

Low-energy $(\lesssim 10 \mathrm{MeV})$ electron beams are conventionally produced in photoemission electron sources based on radio frequency (rf) guns or "photoinjectors." The final bunch length downstream of a photoinjector is dictated by the initial parameters including the photocathode-laser pulse duration, transverse spot size, the electric-field amplitude in the gun cavity and its phase relative to the laser. Typically, bunch lengths on the order of picoseconds are commonly produced in $L$ - and $S$-band rf guns. Shortening these bunches or producing trains of sub-ps microbunches is appealing to a variety of applications including ultrafast electron diffraction [1,2], coherent accelerator-based, e.g., THz light sources [3,4], and injectors for short-wavelength advanced-accelerator concepts $[5,6]$.

To date, bunch compression to produce kA peak currents is often realized after acceleration to $\gtrsim 100 \mathrm{MeV}$ by employing dispersive sections arranged as, e.g., magnetic chicanes [7]. Alternative methods to shorten a relativistic bunch also include velocity bunching [8-11], and ballistic bunching using an accelerating cavity operating at zero crossing. The latter method demonstrated bunching at the sub-100-fs time scale [12] and could possibly produce shorter temporal structures [13]. Similar methods have been extended to the mm-wave regime, e.g., by coupling laserproduced $\mathrm{THz}$ pulses to the beam using undulators [14] or dielectric waveguides [15].

Published by the American Physical Society under the terms of the Creative Commons Attribution 3.0 License. Further distribution of this work must maintain attribution to the author(s) and the published article's title, journal citation, and DOI.
In addition, several techniques have demonstrated narrow-band $\mathrm{THz}$ radiation generation with photoinjector beams by coupling a density-modulated bunch with electromagnetic-radiation mechanisms [16-20]. Among these techniques, two of them are based on impressing a density modulation using a temporally modulated photocathode-laser pulse [19-21]. The use of such a temporally modulated laser was also experimentally shown to support the formation of short-current spikes via wave breaking seeded by nonlinear longitudinal space-charge effects [22].

Most recently, a technique to produce train of microbunches based on a dielectric-lined waveguide (DLW) was realized in a $\sim 70 \mathrm{MeV}$ accelerator [23-25]. In the latter experiment a density modulation was produced using a small chicane to provide the longitudinal dispersion necessary to convert the energy modulation imparted by the beam selfinteraction with its short-range wakefield in the DLW structure.

In this paper, we propose a simple method extending the mechanism proposed in Ref. [23] to low-energy beams. In our configuration a $\sim 5-10 \mathrm{MeV}$ ps-duration beam is energy modulated as it passes through a DLW and ballistically bunched in a subsequent drift. Our approach is similar to the bunching technique commonly used in klystrons [26,27]. Owing to the low intrinsic energy spread typically achieved in photoinjectors, final beam currents in excess of kA's could be produced.

\section{BALLISTIC COMPRESSION FROM WAKEFIELD-INDUCED ENERGY MODULATIONS}

A feature critical to the production of density modulated beams is the capability to produce the required large local longitudinal-phase-space (LPS) chirps via the selfwakefield in the considered DLW structure. We investigate 
this point with a cylindrically symmetric DLW consisting of a hollow dielectric cylinder with inner and outer radii $a$ and $b$ [28], and relative electric permittivity $\varepsilon_{r}$. The outer surface of the dielectric is metallized. We consider the axial longitudinal wake function modes supported by such a structure to be of the form $[29,30]$

$$
w_{z, m}(\zeta)=\kappa_{m} \cos \left(k_{m} \zeta\right),
$$

where $\zeta$ is the position of the observer charge referenced with respect to the source electron and $\kappa_{m}$. [with units of $\mathrm{V} /(\mathrm{mC})]$ and $k_{m}$ are respectively the loss factor and wave vector associated to the $m$ mode supported by the DLW structure. The mode parameters $\kappa_{m}$ and $k_{m}$ are obtained following the methodology described in Ref. [28] by numerically solving the dispersion equation.

An example of a computed Green's function for a structure with parameters $a=400 \mu \mathrm{m}, b=450 \mu \mathrm{m}$, and $\varepsilon_{r}=5.7$ (corresponding to diamond) appears in Fig. 1. The Green's function converges after the inclusion of four modes (the $50 \mu \mathrm{m}$ thickness of the structure supports multiple modes with significant axial fields).

Note that the field in Eq. (1) and the wake function have no dependence on the transverse coordinates. The expected change in longitudinal momentum for a particle within and behind a bunch with line-charge current distribution $\Lambda(z)$ is obtained from the convolution integral

$$
\Delta E(z) \simeq c \Delta p_{z}(z)=L_{d l w} \int_{-\infty}^{z} d z^{\prime} \Lambda\left(z-z^{\prime}\right) w_{z}\left(z^{\prime}\right),
$$

where $L_{d l w}$ is the length of the DLW structure and $z$ the longitudinal coordinate within the bunch.

In contrast with an energy modulation imparted by external fields (e.g., from lasers or rf cavities), the

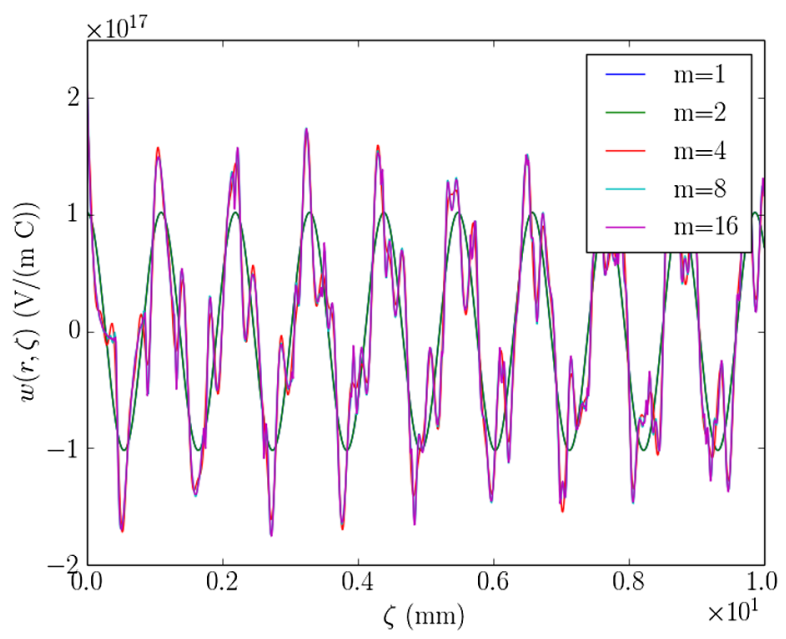

FIG. 1. Wake function computed as $w_{z}(\zeta)=\sum_{l=1}^{m} w_{z, l}$ for $m=1,2,4,8$, and 16 for a DLW structure with parameters $a=400 \mu \mathrm{m}, b=450 \mu \mathrm{m}$, and $\varepsilon_{r}=5.7$. The fundamental-mode (blue trace) wavelength is $\lambda_{1} \simeq 1.09 \mathrm{~mm}$. modulation imparted via wakefields depends on the longitudinal bunch shape. In particular, given the selected parameters for the DLW structure, one should ideally select an electron-bunch distribution with spectral contents capable of exciting the mode(s) supported by the structure; see Fig. 2.

In order to illustrate the proposed concept we elaborate a simple model based on the ideal case of a line-charge electron bunch with a parabolic charge-density profile $\Lambda(z)=\left[3 Q /\left(2 a^{3}\right)\right]\left(a^{2}-z^{2}\right)$ for $|z| \leq a$, where $Q$ is the total bunch charge and $a$ the half width of the distribution; see Fig. 3(a). The corresponding change in energy along the bunch is given by

$$
\begin{aligned}
\Delta E(z) \simeq & \sum_{m=1}^{+\infty} \mathcal{E}\left\{\sin \left[k_{m}(z+a)\right]\right. \\
& \left.-k_{m} a \cos \left[k_{m}(z+a)\right]+k_{m} z\right\},
\end{aligned}
$$

where $\mathcal{E} \equiv \frac{3 \kappa_{m} L_{d l w} Q}{2 k_{m}^{3} a^{3}}$. Considering only the fundamental mode $(m=1)$ and assuming a "cold" initial LPS with no correlation so that $\left(z_{i}, \delta_{i}=0\right)$ (for all $i$ ), where $z_{i}$ and $\delta_{i}$ are respectively the axial coordinate and fractional momentum spread associated to the $i$ th electron, the final fractional momentum spread downstream of the DLW structure becomes

$$
\delta_{f}\left(z_{f}\right) \simeq \frac{E_{i}+\Delta E\left(z_{f}\right)}{E_{f}}-1,
$$

where $E_{i}$ is the bunch's initial mean energy, $E_{f} \equiv E_{i}+$ $1 / Q \int \Delta E(z) \Lambda(z) d z$ its final mean energy, and $z_{f}=z_{i}$.
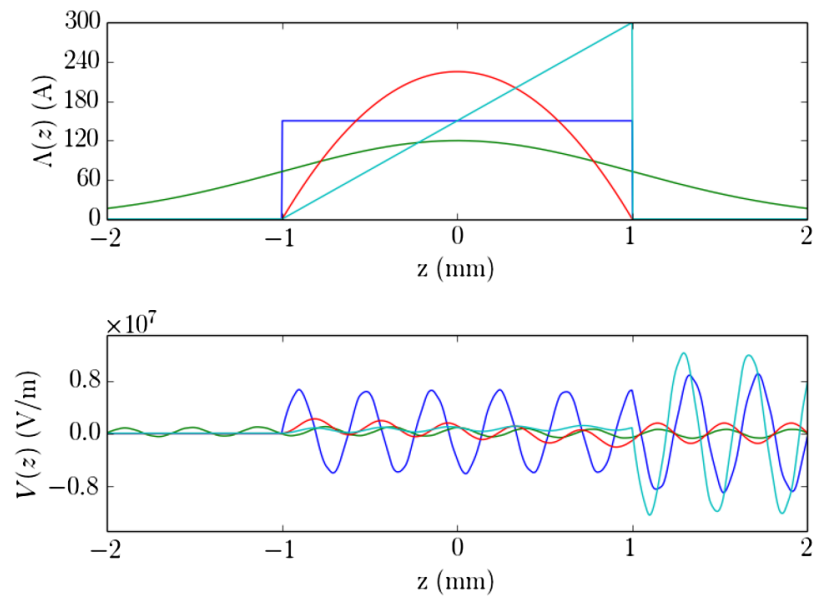

FIG. 2. Charge distributions (top) and corresponding wake potential (bottom) for the same structure parameters as shown in Fig. 1 and for a $1 \mathrm{nC}$ Gaussian bunch with variance (Gaussian distribution) or hard-edge half size (other distributions) of $1 \mathrm{~mm}$. The green, blue, red, and turquoise traces respectively correspond to the case of a Gaussian, parabolic, uniform, and linearly ramped current distributions. The head of the bunch is at $z \leq 0$. 
For the case of short modulation $k a \gg 1$ the final energy can be approximated as $E_{f} \simeq E_{i}-\frac{3 L_{\mathrm{dow}} \kappa_{1} Q}{2 a^{4} k_{1}^{4}} \simeq E_{i}$.

After a section with longitudinal dispersion $R_{56}$, the energy modulation induces a density modulation and the final longitudinal coordinate of an electron is mapped as $z_{d}=z_{f}+R_{56} \delta_{f}$ under a linear single-particle dynamics approximation.

We first consider the case when the root-mean-square (rms) bunch length satisfies $\sigma_{z, i} \equiv\left\langle z_{i}^{2}\right\rangle^{1 / 2} \gtrsim \lambda_{1} \equiv 2 \pi / k_{1}$ so that an energy modulation along the bunch can be impressed; Fig. 3(b) (red trace). In such a case the second term in Eq. (3) dominates the short-wavelength modulation structure and the final longitudinal coordinate is approximately given by

$$
z_{d} \simeq z_{i}-\frac{R_{56} \mathcal{E}}{E_{i}} \cos \left[k_{1}\left(z_{f}+a\right)\right]
$$

At the zero-crossing locations, i.e., the locations along the bunch $z_{f, n}$ such that $\delta_{f}\left(z_{f, n}\right) \propto \cos \left[k_{1}\left(z_{f, n}+a\right)\right]=0$, the local LPS correlation is given by

$$
\left.\mathcal{C} \equiv \frac{d \delta_{f}}{d z_{f}}\right|_{z_{f, n}} \simeq \frac{k_{1} \mathcal{E}}{E_{i}}
$$

The maximum bunching occurs at these zero-crossing points when the following beam line provides a longitudinal dispersion $R_{56}=-\frac{1}{\mathcal{C}}$. The characteristic length of the microbunches formed is approximately given by $\sigma_{z} \simeq R_{56} \tilde{\sigma}_{\delta}$, where $\tilde{\sigma_{\delta}}$ is the uncorrelated (or slice) $\mathrm{rms}$ fractional momentum spread. The microbunches'
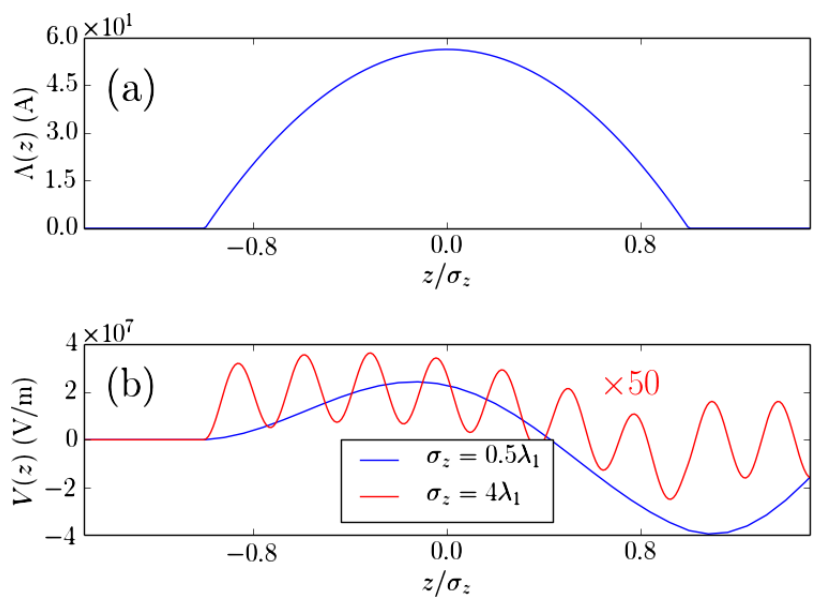

FIG. 3. Charge distributions (a) and corresponding wake potential (b) for two cases of ratio between the rms bunch length $\sigma_{z}$ and fundamental-mode wavelength $\lambda_{1}$. The DLW structure parameters are identical to one used in Fig. 1. The head of the bunch corresponds to $z \leq 0$. The wake potential associated to the $\sigma_{z}=0.5 \lambda_{1}$ case is scaled by a factor 50 for clarity. separation is $\Delta z \equiv z_{f, n}-z_{f, n-1} \simeq \lambda_{1}$ for an incoming beam with vanishing correlated energy spread.

At relativistic energies, the longitudinal dispersion $R_{56}$ necessary to form the microbunches is often provided by a dispersive section, e.g., a bunch-compressor chicane [7] as accomplished in Ref. [23]. Here we note that at energies below $\sim 10 \mathrm{MeV}$ (non-ultra-relativistic regime), the large LPS slope resulting from the large axial fields supported in a DLW requires a relatively small $R_{56}$ that can be readily produced by a drift space. A drift with length $D$ has a longitudinal dispersion,

$$
R_{56} \simeq-\frac{D}{\gamma^{2}},
$$

where $\gamma$ is the bunch's Lorentz factor and we take $\beta \equiv\left(1-1 / \gamma^{2}\right)^{1 / 2} \simeq 1$ for simplicity.

Practically, for a $\sim 5 \mathrm{MeV}$ electron bunch passing through a 10-cm long DLW structure capable of supporting $\sim 0.5 \mathrm{MV} / \mathrm{m}$ peak field a "local" chirp $\mathcal{C} \simeq 10^{3} \mathrm{~m}^{-1}$ can be obtained for a $0.5 \mathrm{~mm}$ modulation wavelength. The corresponding local density spike could form via ballistic bunching after a drift of length below $D \leq 1 \mathrm{~m}$. The expected modulation amplitude $\sim 0.5 \mathrm{MeV}$ is much larger than the typical uncorrelated energy spread of a few $\mathrm{keV}$ routinely achieved in $\mathrm{rf}$ guns [32,33]. Additionally, the relatively low $R_{56}$ and small uncorrelated energy spread are also beneficial to the production of very short $(<100 \mathrm{fs})$ density spikes. This simple estimate motivates further investigation of the scheme using a bunch generated by a conventional photoemission electron gun.

In addition, furthering our point about the dependence of the energy modulation on bunch shape, we now examine the case when the rms bunch length fulfills $\sigma_{z, i} \simeq \lambda_{1} / 2$; see Fig. 3(b) (blue trace). In this regime, the induced energy change along the bunch produces an energy depression between the head and tail of the bunch and has the proper sign to be compressed via ballistic bunching. Although the introduced chirp is nonlinear, it can eventually lead to the production of a high peak current. This approach, however, only bunches a fraction of the bunch and actually debunches the head of the bunch. Despite this drawback, this scheme is appealing given its simplicity and absence of need for a precisely synchronized external field as used in ballistic bunching using a buncher cavity [12]. This passive bunching method is therefore inherently self-synchronized and in principle not subject to time jitter (the main source of jitter is associated to charge fluctuations that impact the imparted energy modulation and could consequently result in shot-to-shot fluctuations of the peak current).

Finally, it should be pointed out that higher-order (e.g., dipole) modes can also affect the bunch transverse dynamics but are neglected in the present treatment as we assume the bunch is cylindrical symmetric and axially centered on the DLW axis. Given the short length of the DLW 
considered in the remainder of the paper, possible detrimental effects on the transverse beam dynamics can be practically corrected, e.g., by mounting the DLW structure on translational stages.

\section{NUMERICAL MODELING AND ANALYSIS}

To explore the possibilities discussed in the previous section we perform beam-dynamics simulations. The numerical simulations are carried with the beam-dynamics program ASTRA [34] which takes into account space-charge effects using a cylindrical-symmetric quasistatic spacecharge algorithm. The beam-DLW interaction is modeled via the Green's function approach briefly outlined above and detailed in Ref. [35]. The Green's function employed in our simulations throughout this paper is constructed using the wake associated to the four modes of the considered structure as supported by Fig. 1.

In most of our simulations the electron bunch is modeled as an ensemble of 100000 macroparticles. The grid used to bin the macroparticle distribution within the cylindricalsymmetric space-charge algorithm is typically set up with a number of radial and longitudinal bins $n_{r}=11$ and $n_{z}=500$. The large number of longitudinal bins ensures a temporal resolution at the sub-50-fs scale is realized for most of the current distribution generated throughout this paper. We note however, that binary collisions (Boersch effect [36]) are not taken into account in the mean-field space-charge algorithm employed in ASTRA and might result in a degradation of the peak current quoted in this paper.

To characterize the temporal structure of the bunch, we represent the macroparticles' temporal distribution as $\Lambda(z)=\frac{1}{N} \sum_{i=1}^{N} \delta\left(z-z_{i}\right)$ and compute the bunch form factor (BFF) $\tilde{F}(\omega)=\left|1 /(2 \pi) \int_{-\infty}^{+\infty} \Lambda(z / c) e^{-i \omega t}\right|^{2}$ as

$$
\tilde{F}(\omega)=\frac{1}{N^{2}}\left(\left|\sum_{i}^{N} \cos \frac{\omega z_{i}}{c}\right|^{2}+\left|\sum_{i}^{N} \sin \frac{\omega z_{i}}{c}\right|^{2}\right)
$$

where $N$ is the number of macroparticles used in the simulation. The BFF is commonly used to characterize the performance of the accelerator-based radiation source [37]. We note that in some cases, e.g., for the production of shortwavelength coherent radiation, transverse suppression effects might be prominent and should be properly accounted for by utilizing a three-dimensional expression for the BFF; see, e.g., Ref. [38].

\section{A. Subpicosecond bunch train formation}

We first investigate the practical realization of the scheme described in Sec. II to produce trains of subpicosecond bunches and to demonstrate the versatility of the method, we consider two examples of implementation. The generic setup consists of an rf-gun electron source followed by a DLW as diagrammed in Fig. 4. Downstream of the

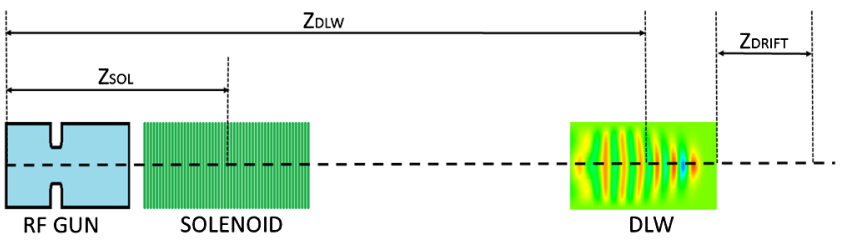

FIG. 4. Overview of the photoinjector setup used for the numerical simulations. The distances $Z_{S O L}$ and $Z_{D L W}$ correspond respectively to the location of the center of the solenoid and DLW structure referenced to the photocathode surface, and $Z_{\text {drift }}$ represents the drift distance downstream of the DLW structure necessary for ballistic bunching.

DLW the beam is focused with a second solenoid, e.g., to produce a waist at the location a transition-radiation target. The rf gun is taken to be an $S$-band $(2.856 \mathrm{GHz}) 1 / 2$-cell cavity similar to the one currently in use at the linac coherent light source (LCLS) [39]. Similar results are then confirmed using a $1 / 2$-cell $L$-band $(1.3 \mathrm{GHz})$ gun similar to the one used at the FLASH facility in DESY [40].

The photocathode-laser distribution was chosen to follow a plateau temporal distribution and its transverse size along with the location of the DLW, and solenoid strength were optimized using a multiobjective optimizer [41] to maximize beam transmission through the structure and minimize the transverse beam size at the DLW center. The list of optimized operating parameters are gathered in Table I ("S-band" column). We note that the choice of the DLW parameters is a compromise between modulation wavelength $\lambda_{1}$, energy modulation amplitude-which affects the bunching length-and beam transmission. For example, a shorter DLW structure relaxes the requirements

TABLE I. Beam line settings and DLW-structure parameters used in the ASTRA simulations. The beam line configuration with some of the associated parameters is depicted in Fig. 4.

\begin{tabular}{lccc}
\hline \hline & $S$ band & $L$ band & \\
\hline Parameter & & & units \\
\hline Laser pulse rms duration & 3 & 7 & $\mathrm{ps}$ \\
Laser pulse rise time & 100 & 100 & $\mathrm{fs}$ \\
Laser rms spot size & 0.72 & 1.1 & $\mathrm{~mm}$ \\
Initial charge & 1 & 1 & $\mathrm{nC}$ \\
Peak field on cathode & 120 & 34 & $\mathrm{MV} / \mathrm{m}$ \\
Solenoid 1 position & 0.20 & 0.0 & $\mathrm{~m}$ \\
Solenoid 1 strength & 0.26 & 0.17 & $\mathrm{~T}$ \\
Solenoid 2 position & 1.35 & 1.0 & $\mathrm{~m}$ \\
Solenoid 2 strength & 0.45 & 0.15 & $\mathrm{~T}$ \\
DLW position & 0.9 & 0.34 & $\mathrm{~m}$ \\
DLW inner radius $(a)$ & 350 & 500 & $\mu \mathrm{m}$ \\
DLW outer radius $(b)$ & 363 & 550 & $\mu \mathrm{m}$ \\
DLW length & 11 & 4 & $\mathrm{~cm}$ \\
DLW fund. frequency $f_{1}$ & 1000 & 400 & $\mathrm{GHz}$ \\
Transmission through DLW & 85 & 98 & $\%$ \\
Average kinetic energy & 6.1 & 3.8 & $\mathrm{MeV}$ \\
\hline \hline
\end{tabular}


on beam sizes and emittances at the structure, but necessitates a longer drift to bunch the beam (as the amplitude of the imparted energy modulation is smaller than for a longer structure). Additionally, the number of potential microbunches depends on the incoming bunch length and $\lambda_{1}$. For example, a Gaussian bunch with rms length $\sigma_{z}$ will typically result in the formation of $N_{b} \sim 4 \sigma_{z} / \lambda_{1}$ microbunches. Additionally, varying $\sigma_{z}$ for a given bunch charge and fundamental-mode wavelength $\lambda_{1}$ affects the initial peak current and consequently the amplitude of the imparted energy modulation as inferred from Eq. (4).

We present, for the $S$-band case listed in Table I, the evolution of the BFF over a frequency range $f \equiv \frac{\omega}{2 \pi} \in[0.5,3.5] \mathrm{THz}$ as a function of the drift distance from the DLW exit $\left(z_{\text {drift }}\right)$ in Fig. 5(a). The corresponding longitudinal-density evolution appears in Fig. 5(b). For this set of parameters, ten microbunches are produced and a maximum bunching of $\tilde{F}\left(\omega_{1}\right) \simeq 0.20$ is obtained at the DLW fundamental mode's wavelength $\lambda_{1} \simeq 382 \mu \mathrm{m}$. In addition, harmonics of the fundamental mode $f_{1, n}=n f_{1}$ are observed. For the selected DLW parameters and the corresponding thin dielectric layer, only the fundamental mode significantly influences the bunch dynamics.

The current and LPS distributions at the DLW exit and at the location of maximum bunching (at $s \simeq 1.30 \mathrm{~m}$ from the photocathode) appear in Fig. 6. Peak currents on the order of $1 \mathrm{kA}$ are achieved for a beam with mean momentum of $\left\langle p_{z}\right\rangle \simeq 6.12 \mathrm{MeV} / c$. The shortest current spike generated has a full-width half-maximum (FWHM) duration of
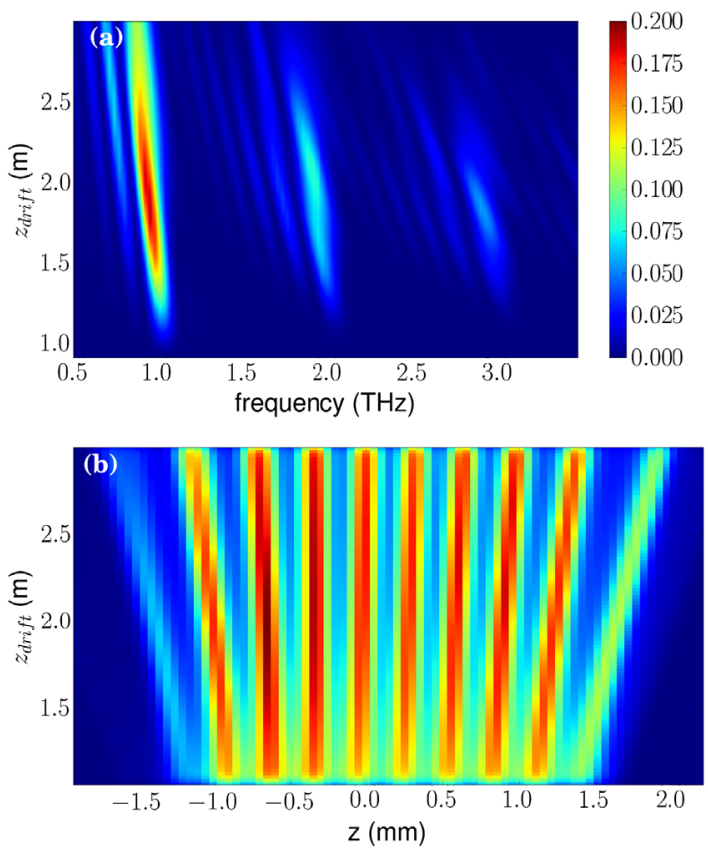

FIG. 5. Bunch form factor (BFF) (a) and bunch longitudinal density (b) evolution as a function of the drift length referenced with respect to the DLW exit. The simulations correspond to the parameters listed under the S-band column in Table I. $\sim 30$ fs. These results are comparable to the ones experimentally obtained through wave breaking in Ref. [22] albeit with a much higher contrast ratio [42]. The origin of the nonuniform bunching across the beam with peak-topeak variation in the microbunch current is twofold. First, the slice-energy-spread positional variation along the bunch affects the shortest structure achievable at a given location. Second, the LPS prior to the DLW has initial correlations [as seen on the blue density plotted in Fig. 6(b)] which affect the bunching uniformity across the microbunches. This latter initial correlation is also responsible for the apparent "walk-off" feature (the microbunches spread apart from each others as they drift) of the microbunches visible in Fig. 5(b). Figure 6(c) indicates strong harmonic content at the second and third harmonic frequencies of $f_{1}$-also observed at the location of maximum bunching.

Moreover, the higher harmonics are limited by the precision of the microbunch spacing within the bunch; a higher frequency DLW will lead to more microbunches which will be more limited by the initial correlated LPS. We can investigate this feature by using a lower frequency structure of $500 \mathrm{GHz}$ in the same context of the $1 \mathrm{THz}$ example illustrated above. The current and LPS is shown in Figs. 7(a) and 7(b) as well as the associated BFF over the frequency range $(0.25 \mathrm{THz}, 10 \mathrm{THz})$, as shown in Fig. 7(c) for maximum compression (red trace). The very strong higher harmonic content is notably due to larger and more
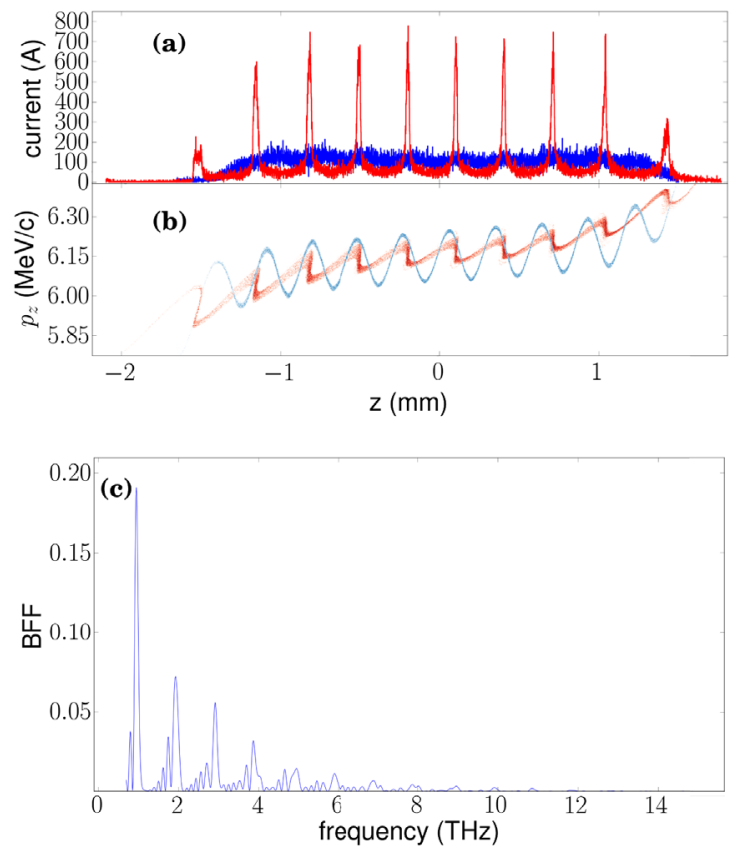

FIG. 6. Current profiles (a) and associated longitudinal phase spaces (LPS) (b) simulated at the exit of the DLW (blue trace) and at the location of maximum bunching (red trace) $z=1.3 \mathrm{~m}$ from the photocathode. Bunch form factor (BFF) (c) obtained at $z=1.3 \mathrm{~m}$ from the photocathode. The simulations correspond to the parameters listed under the S-band column in Table I. 

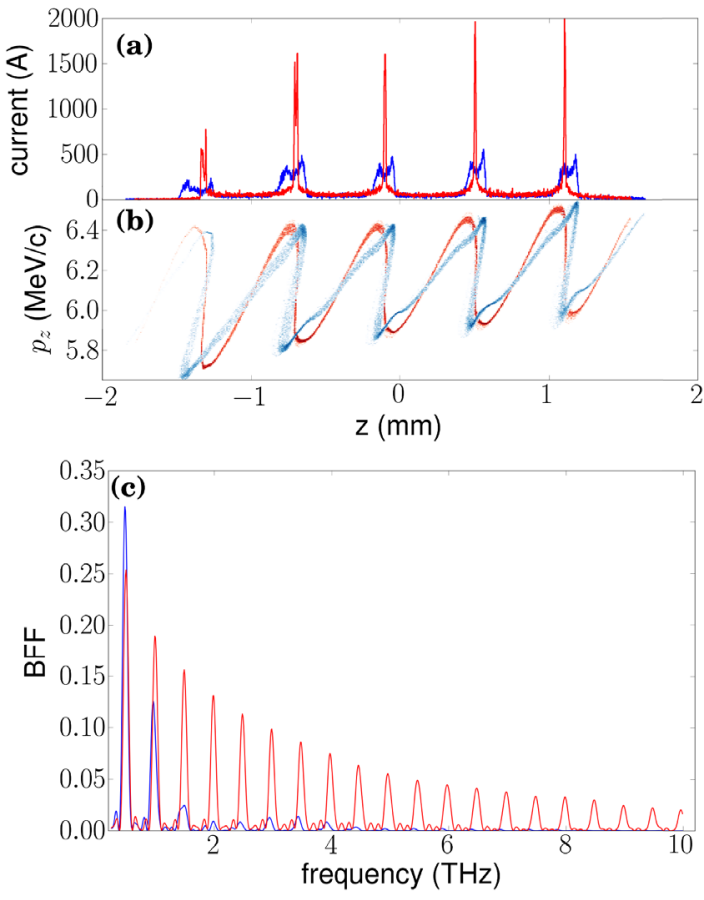

FIG. 7. Current profiles (a) and associated longitudinal phase spaces (LPS) (b) simulated at maximum compression $31 \mathrm{~cm}$ downstream of the DLW (red traces) and at the location of slight overcompression $52 \mathrm{~cm}$ downstream of the DLW. Bunch form factor (BFF) (c) obtained at the similar locations. The simulations correspond to the parameters listed under the S-band column in Table I with the exception of the geometric parameters of the DLW structure selected to be $a=350 \mu \mathrm{m}$, and $b=393 \mu \mathrm{m}$.

precisely spaced microbunches. Additionally, we may want to suppress higher harmonics or amplify the fundamental; this could easily be done by selecting a bunch which is undercompressed or overcompressed such that the microbunches span a larger spatial extent; see Figs. 7(a), 7(b), and 7 (c) (blue trace).

Finally, the evolution of the transverse beam sizes and emittance is respectively shown in Figs. 8(a) and 8(b) for the case presented in Fig. 6. The addition of a second solenoid at $s \simeq 1.2 \mathrm{~m}$ can transversely focus the beam down to $\sigma_{x}=\sigma_{y} \simeq 45 \mu \mathrm{m}$ at an axial location close to the maximum bunching; see Fig. 8(c). The simulated small rms beam size confirms that the one-dimensional BFF approach adopted earlier can accurately be used to estimate the properties of radiation emitted at wavelengths $\lambda \gg \gamma^{-1} \sigma_{x, y} \sim 5 \mu \mathrm{m}$. It is therefore applicable to the $\mathrm{THz}$ regime. The small transverse size could also permit the use of a second DLW as a narrow-band $\mathrm{THz}$ radiator as explored in Ref. [43].

The location of maximum bunching depends primarily on the wakefield amplitude compared to the average bunch energy. Operating the rf gun at higher peak fields leads to larger ballistic bunching lengths downstream of the DLW structure and vice versa. Alternatively, shorter bunching lengths can be achieved by decreasing the bunch length at

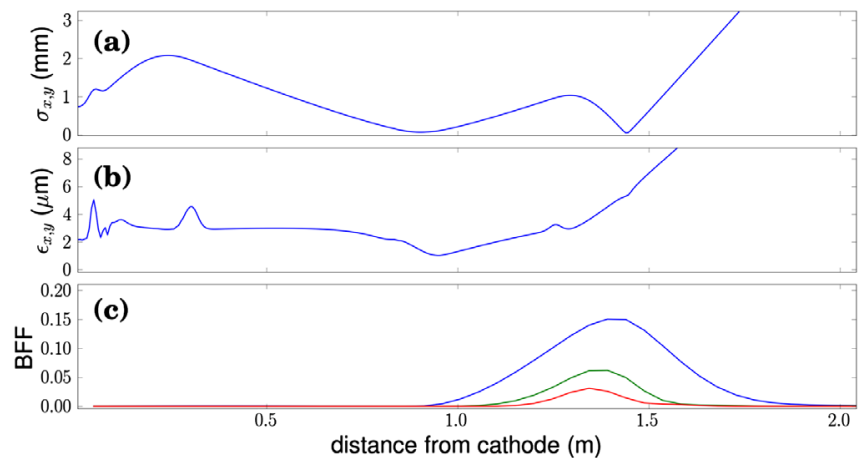

FIG. 8. Transverse horizontal $\sigma_{x}$ and vertical $\sigma_{y}$ rms beam sizes (a), corresponding transverse emittances (b) and bunch form factor (BFF) (c) evolution along the beam line. The BFF is evaluated at $f_{1}=1 \mathrm{THz}$ (blue trace) and at the second (green trace) and third (red trace) harmonics. The simulations correspond to the parameters listed under the $S$-band column in Table I.

the cost of a lower number of microbunches. To confirm the applicability of our concept to other configurations we carried a similar study as the one presented above for the case of an L-band rf gun.

For this case we consider the setup available at the Fermilab's A0 photoinjector [44] which incorporates a first-generation L-band gun used at the decommissioned Tesla-test facility at DESY [45]. The gun is nested in three solenoidal lenses. An optimization similar to the one carried for the S-band case was conducted and the resulting operating parameters are displayed in Table I ( $L$-band column). For completeness the BFF and longitudinal density evolution downstream of the DLW are shown in Fig. 9. As in the S-band case we observe strong bunching at the DLW fundamental mode's frequency (in this case $\lambda_{1} \simeq 750 \mu \mathrm{m}$ as the DLW parameters are different). But in contrast with the $S$-band case the higher-harmonic content of the BFF are significantly suppressed. The change in the fundamental frequency as the bunch drifts downstream of the DLW appears stronger than for the $S$-band case and is due to a more prominent walk-off effect due to the lower beam energy.

\section{B. Passive bunching and shaping}

We now turn to another potential application of the scheme detailed in Sec. II to bunch or shape an electron beam produced via photoemission from an $\mathrm{rf}$ gun (this corresponds to the case when $\sigma_{z} \lesssim \lambda_{1}$ ).

To illustrate our point, we first consider the case of the $L$-band gun just discussed in the previous section and instead of using the DLW parameters of Table I, we consider a structure with inner radius $a=650 \mu \mathrm{m}$ to produce a global correlated energy spread as the fundamental-mode wavelength of the DLW becomes comparable to the bunch length. As mentioned earlier, the inherent nonlinear LPS distortion exhibits a correlation between the 

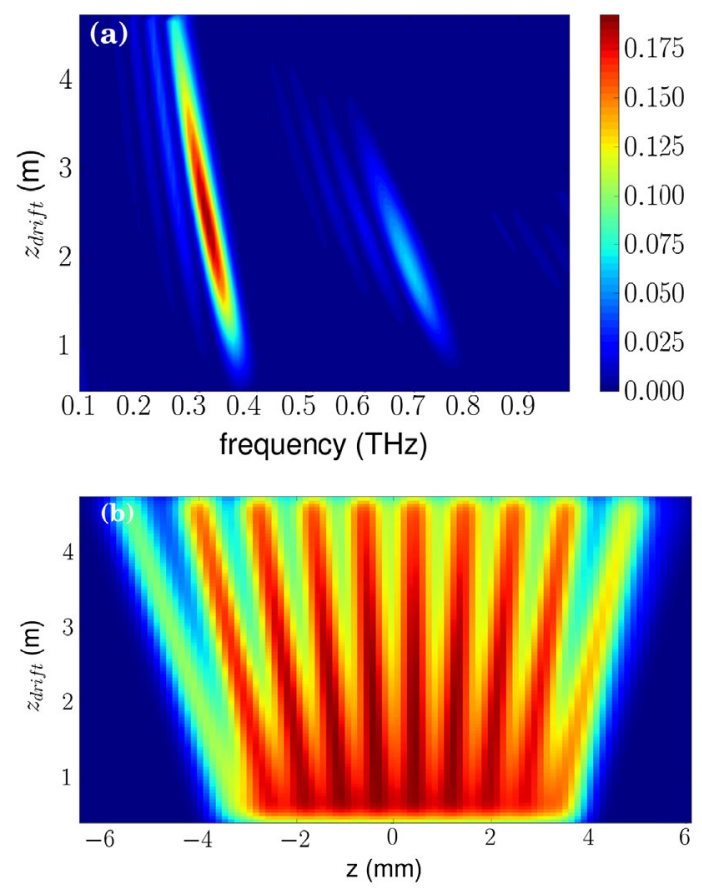

FIG. 9. Bunch form factor (BFF) (a) and bunch longitudinal density (b) evolution as a function of the drift length referenced with respect to the DLW exit. The simulations correspond to the parameters listed under the " $L$-band" column in Table I.

depleted energy location and tail that has the proper sign for compression via ballistic bunching.

We exemplify this possibility by exploring the change in the maximum peak current downstream of a DLW structure with different dielectric thicknesses. The DLW is chosen to have a fixed inner radius $a=650 \mu \mathrm{m}$ and the mode's wavelength is varied with different dielectric thicknesses. Although thicker dielectrics generally lead to a larger population of modes, the Gaussian shape employed in this study mostly excites the fundamental. The results appear in Fig. 10 and indicate that a peak current on the order of $\sim 10 \mathrm{kA}$ is attained when the fundamental-mode wavelength is $\sim 2.06 \mathrm{~mm}$ (corresponding to $\sigma_{z} \simeq \lambda_{1} / 2$ as $\sigma_{z}=1.01 \mathrm{~mm}$ ). It should be pointed out that the quoted currents are most likely overestimated due the absence of collisional effects in the space-charge algorithm implemented in ASTRA. The latter wavelength corresponds to a structure with outer radius $b=855 \mu \mathrm{m}$ (or dielectric thickness $\tau \equiv b-a=205 \mu \mathrm{m})$. The associated current profiles and LPS appear in Fig. 11 and illustrate the role of the initial longitudinal emittance of the bunch before the DLW (i.e., the maximum peak current is achieved for an initial axial slice with smallest slice energy spread). In Fig. 11 only $7.1 \%$ of the population resides within the current spike while the rest contributes to the formation of longitudinal tails. This low-current population of the bunch could in principle be reduced by exploring some energytransverse correlations in conjunction with transverse

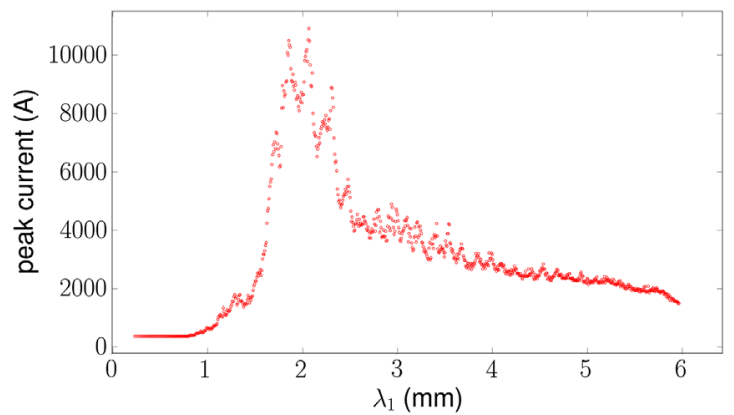

FIG. 10. Maximum peak current as a function of the fundamental-mode wavelength $\lambda_{1}$. The observed noise comes from numerical errors in precisely determining the value of the axial position where the peak current is maximized. These simulations are carried with the beam parameters summarized in Table I $L$-band column but for a DLW structure with inner radius $a=650 \mu \mathrm{m}$. The fundamental-mode wavelength is varied by changing the structure outer radius $b$.

collimators. Also, due to the relatively large inner radii needed to support wavelengths comparable to the bunch length, this technique can in principle easily be scaled to higher bunch charges. Finally, we note that the current profiles shown in Fig. 11 can actually find applications, e.g., to investigate wakefield effects in accelerating structures [46] and in compact beam-driven acceleration schemes utilizing low-energy drive bunches.

As a final application we investigate the possibility of producing low-energy bunches with linearly ramped current profiles. This type of distribution is sought after to improve the transformer ratio- the maximum accelerating wakefield over the decelerating field experienced by the driving bunch - in collinear beam-driven acceleration schemes [47]. We demonstrate that a standard distribution typically produced downstream of an $\mathrm{rf}$ gun can be

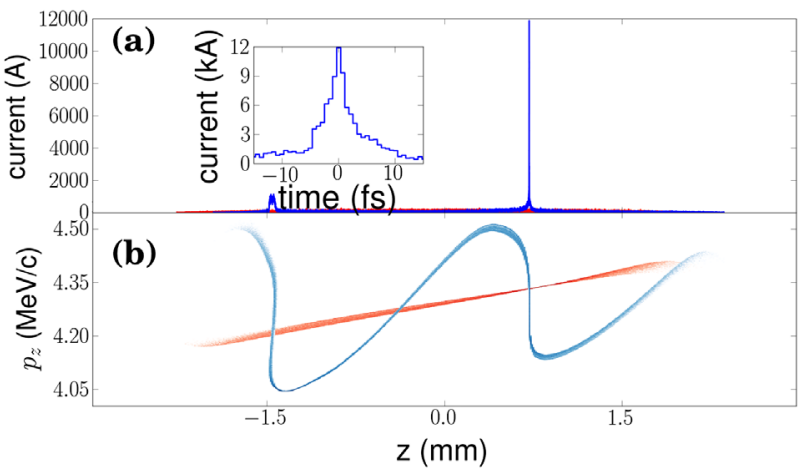

FIG. 11. Current profiles (a) and longitudinal phase spaces (LPS) (b) at the entrance of the DLW structure (red traces) and at location of maximum compression (blue traces). The simulation corresponds to the case $\lambda=2.06 \mathrm{~mm}$ in Fig. 10. The inset in plot (a) corresponds to a zoom of plot (a) around the $\sim 12 \mathrm{kA}$ peak with its origin of the temporal axis corresponding to $z=0.693 \mathrm{~mm}$ in plot (a) axial coordinate. Maximum bunching, in this scenario, occurs $43.9 \mathrm{~cm}$ downstream of the DLW. 


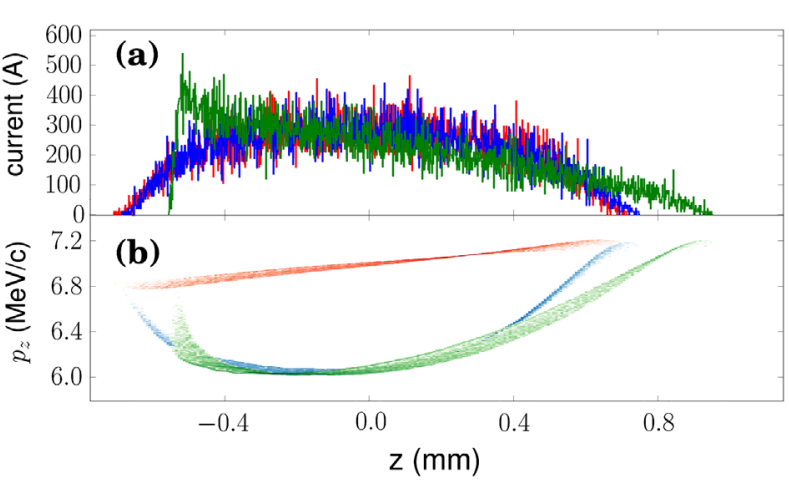

FIG. 12. Current profiles (a) and longitudinal phase spaces (b) at the entrance (red traces) and exit (red traces) of the DLW structure and $0.2 \mathrm{~m}$ downstream of the structure $(s \simeq 0.54 \mathrm{~m}$ from the photocathode surface) where a quasilinear current profile is achieved (green traces).

transformed into a ramped bunch with quasilinear dependency on $z$. We take the example of the S-band gun considered in Sec. III A and set $L / \lambda_{1} \approx 1 / 2$ where $L$ is the full longitudinal size of the bunch upstream of the DLW structure. For these simulations, the axial-field amplitude at the cathode is set to $E_{0}=140 \mathrm{MV} / \mathrm{m}$. Such an increase (compared to the set of parameters displayed in Table I) was required to mitigate bunch lengthening. Figure 12 depicts the LPS evolution and associated current profiles associate to the bunch as it enters (red trace), exits (blue trace) the DLW and after a drift of $0.2 \mathrm{~m}$ (green trace). The interplay of the DLW wakefield and longitudinal-space-charge force results in the appearance of nonlinear correlations in the LPS. These nonlinearities provide some control over the current profile.

To quantify the performance of the current profile simulated in Fig. 12(b) (green trace), we compute its wakefield in a DLW with inner and outer radii respectively $a=165 \mu \mathrm{m}, b=197 \mu \mathrm{m}$ and the relative dielectric permittivity is kept to $\varepsilon_{r}=5$.7. The resulting wakefield behind the bunch has a peak accelerating field amplitude of $E^{+} \simeq 60 \mathrm{MV} / \mathrm{m}$; see Fig. 13. The transformer ratio is numerically inferred as $\mathcal{R} \equiv\left|E_{+} / E_{-}\right|$, where $E_{-} \simeq$ $8.2 \mathrm{MV} / \mathrm{m}$ is the maximum amplitude of the decelerating electric field within the electron bunch. The achieved transformer ratio of $\mathcal{R} \simeq 7.3$ is comparable to the ideal ratio of $\mathcal{R}=n_{p} \pi \simeq 9.4$ predicted for an ideal linearly ramped current profile (here $n_{p} \simeq 3$ is the number of mode wavelength comprised within the total bunch length) [47]. Depending on the desired application, the photoinjector settings and DLW parameters could be adjusted to produce a ramped current profile after further acceleration in a subsequent linac.

Finally, a finer control over the bunch shape could possibly be implemented using several DLW structures with properly selected fundamental-mode wavelengths. Such a multifrequency DLW approach would be an extension of the scheme described in Ref. [48] to higher frequencies.

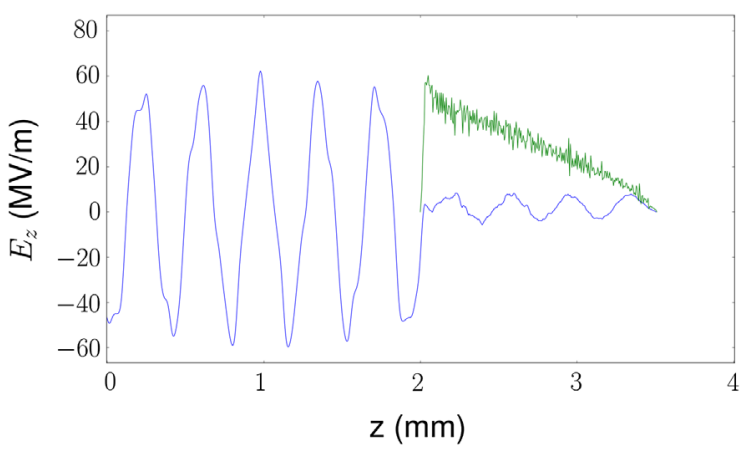

FIG. 13. Longitudinal wakefield (blue trace) produced behind a bunch with the longitudinal distribution (green trace) identical to the one shown in Fig. 12 [plot (a), green trace] for a bunch charge of $1 \mathrm{nC}$. The structure used for the wakefield generation has the geometric parameters $a=165 \mu \mathrm{m}, b=197 \mu \mathrm{m}$ and $\varepsilon_{r}=5.7$.

\section{SUMMARY}

In summary, we presented a relatively simple technique to bunch non-ultra-relativistic beams commonly produced by photoinjectors. The method is shown to support the generation of bunch trains consisting of subpicosecond microbunches. Moreover, we demonstrated that a DLW with a lower-frequency fundamental mode could act as a passive buncher and produce multi-kA bunches. In addition, we discuss the application of the technique to form bunches with linearly ramped current profiles as needed to improve the transformer ratio in beam-driven advancedacceleration techniques. One of the main advantages of the method is that it relies on the bunch interaction with its self-wakefields which are inherently synchronized: the technique is therefore not prone to temporal jitter.

We expect the proposed method to find useful applications that span accelerator-based compact THz-radiation sources, ultrafast electron diffraction and in photoinjectors for short-wavelength linacs.

It is also worth noting that the scheme could in principle be combined with other electron-emission processes (e.g., thermionic- or field-emission) but a detailed exploration is beyond the scope of the present study.

Finally, other wakefield mechanisms, e.g., the use of a corrugated pipe $[49,50]$ could provide an alternative to DLWs and also lead to similar results [51]. Our selection of a DLW structure was mainly motivated by its manufacturing simplicity and wide use in advanced accelerator R\&D.

\section{ACKNOWLEDGMENTS}

This work was supported by the Defense Threat Reduction Agency, Basic Research Award No. HDTRA1-10-1-0051, to Northern Illinois University and by the Department of Energy Contracts No. DE-FG02-08ER41532 and No. DESC0011831 with Northern Illinois University. P. P. is partially supported by DOE Contract No. DE-AC02-07CH11359 to the Fermi research alliance LLC. 
[1] A. H. Zewail and J. M. Thomas, 4D Electron Microscopy: Imaging in Space and Time (Imperial College Press, London, 2010).

[2] R. K. Li, P. Musumeci, H. A. Bender, N. S. Wilcox, and M. Wu, J. Appl. Phys. 110, 074512 (2011).

[3] A. Gover, Phys. Rev. ST Accel. Beams 8, 030701 (2005).

[4] A.-S. Müller, Rev. Accl. Sci. Tech. 03, 165 (2010).

[5] A. Modena, Z. Najmudin, A. E. Dangor, C. E. Clayton, K. A. Marsh, C. Joshi, V. Malka, C. B. Darrow, C. Danson, D. Neely, and F. N. Walsh, Nature (London) 377, 606 (1995).

[6] D. F. Gordon, A. Ting, T. Jones, B. Hazi, R. F. Hubbard, and P. Sprangle, in Proceedings of the 2003 Particle Accelerator Conference, Portland, OR (IEEE, New York, 2003), p. 1846.

[7] B. E. Carlsten and S. M. Russel, Phys. Rev. E 53, R2072 (1996).

[8] X. J. Wang, X. Qiu, and I. Ben-Zvi, Phys. Rev. E 54, R3121 (1996).

[9] X. J. Wang and X. Y. Chang, Nucl. Instrum. Methods Phys. Res., Sect. A 507, 310 (2003).

[10] P. Piot, L. Carr, W. S. Graves, and H. Loos, Phys. Rev. ST Accel. Beams 6, 033503 (2003).

[11] M. Ferrario et al., Phys. Rev. Lett. 104, 054801 (2010).

[12] T. van Oudheusden, P. L. E. M. Pasmans, S. B. van der Geer, M. J. de Loos, M. J. van der Wiel, and O. J. Luiten, Phys. Rev. Lett. 105, 264801 (2010).

[13] K. Flöttmann, Nucl. Instrum. Methods Phys. Res., Sect. A 740, 34 (2014).

[14] C. Sung, S. Ya. Tochitsky, S. Reiche, J. B. Rosenzweig, C. Pellegrini, and C. Joshi, Phys. Rev. ST Accel. Beams 9, 120703 (2006).

[15] L. J. Wong, A. Fallahi, and F. X. Kärtner, Opt. Express 21, 9792 (2013).

[16] P. Muggli, V. Yakimenko, M. Babzien, E. Kallos, and K. P. Kusche, Phys. Rev. Lett. 101, 054801 (2008).

[17] Y.-E Sun, P. Piot, A. Johnson, A. H. Lumpkin, T. J. Maxwell, J. Ruan, and R. Thurman-Keup, Phys. Rev. Lett. 105, 234801 (2010).

[18] P. Piot, Y.-E Sun, T. J. Maxwell, J. Ruan, A. H. Lumpkin, M. M. Rihaoui, and R. Thurman-Keup, Appl. Phys. Lett. 98, 261501 (2011).

[19] Y.-C. Huang, Int. J. Mod. Phys. B 21, 287 (2007).

[20] M. Bolosco, I. Boscolo, F. Castelli, S. Cialdi, M. Ferrario, V. Petrillo, and C. Vaccarezza, Nucl. Instrum. Methods Phys. Res., Sect. A 577, 409 (2007).

[21] Y. Li and K.-J. Kim, Appl. Phys. Lett. 92, 014101 (2008).

[22] P. Musumeci, R. K. Li, and A. Marinelli, Phys. Rev. Lett. 106, 184801 (2011).

[23] S. Antipov, C. Jing, M. Fedurin, W. Gai, A. Kanareykin, K. Kusche, P. Schoessow, V. Yakimenko, and A. Zholents, Phys. Rev. Lett. 108, 144801 (2012).

[24] S. Antipov, M. Babzien, C. Jing, M. Fedurin, W. Gai, A. Kanareykin, K. Kusche, V. Yakimenko, and A. Zholents, Phys. Rev. Lett. 111, 134802 (2013).

[25] S. Antipov, C. Jing, P. Schoessow, A. Kanareykin, B. Jiang, V. Yakimenko, A. Zholents, and W. Gai, AIP Conf. Proc. 1507, 421 (2012).
[26] D. R. Hamilton, J. K. Knipp, and J. B. Horner Kuper, Klystron and Microwave Triodes, MIT Radiation Laboratory Series (McGraw-Hill, New York, 1948).

[27] B. C. Yunn, in Proceedings of the 18th Particle Accelerator Conference, New York, 1999 (IEEE, New York, 1999), p. 2453.

[28] M. Rosing and W. Gai, Phys. Rev. D 42, 1829 (1990).

[29] A. Chao, Physics of Collective Instabilities in High-Energy Accelerators, Wiley Series in Beams \& Accelerator Technologies (John Wiley and Sons, New York, 1993).

[30] G. V. Stupakov, Report No. SLAC-PUB-8683 (unpublished report available from the Stanford Linear Accelerator Center, 2000).

[31] S. Antipov, C. Jing, P. Schoessow, A. Kanareykin, B. Jiang, V. Yakimenko, A. Zholents, and W. Gai, Rev. Sci. Instrum. 84, 022706 (2013).

[32] Z. Huang, D. Dowell, P. Emma, and C. Limborg-Duprey, in Proceedings of the 21st Particle Accelerator Conference, Knoxville, TN, 2005 (IEEE, Piscataway, NJ, 2005), p. 3570.

[33] M. Hüning and H. Schlarb, in Proceedings of the 2003 Particle Accelerator Conference, Portland, OR, edited by J. Chew (Ref. [6]), p. 2074.

[34] K. Flöttmann, ASTRA: A Space Charge Algorithm, User's Manual, available from the World Wide Web at http://www .desy.de/ mpyflo/AstraDokumentation (unpublished).

[35] M. Dohlus, K. Flöttmann, and C. Henning, arXiv:1201.5270; Report No. DESY 12-02 (available from DESY Hamburg, Germany).

[36] H. Boersch, Z. Phys. 139, 115 (1954).

[37] J. S. Nodvick and D. S. Saxon, Phys. Rev. 96, 180 (1954).

[38] E. Saldin, E. Schneidmiller, and M. Yurkov, Nucl. Instrum. Methods Phys. Res., Sect. A 539, 499 (2005).

[39] D. T. Palmer, R. H. Miller, H. Winick, X. J. Wang, K. Batchelor, M. Woodle, and I. Ben-Zvi, in Proceedings of the Particle Accelerator Conference, Dallas, TX, 1995 (IEEE, New York, 1995), p. 982.

[40] B. Dwersteg, K. Flöttmann, J. Sekutowicz, and Ch. Stolzenburg, Nucl. Instrum. Methods Phys. Res., Sect. A 393, 93 (1997).

[41] M. Borland and H. Shang (private communication).

[42] P. Musumeci, R. K. Li, K. G. Roberts, and E. Chiadroni, Phys. Rev. ST Accel. Beams 16, 100701 (2013).

[43] A. M. Cook, R. Tikhoplav, S. Y. Tochitsky, G. Travish, O. B. Williams, and J. B. Rosenzweig, Phys. Rev. Lett. 103, 095003 (2009).

[44] J.-P. Carneiro, N. Barov, H. Edwards, M. Fitch, W. Hartung, K. Floettmann, S. Schreiber, and M. Ferrario, Phys. Rev. ST Accel. Beams 8, 040101 (2005).

[45] J. Andruszkow et al. (TESLA Collaboration), Phys. Rev. Lett. 85, 3825 (2000).

[46] C. Adolphsen et al., Next Linear Collider (NLC) Newsletter 1, 2 (2000) (unpublished report available from the Stanford Linear Accelerator Center, 2000).

[47] K. L. Bane, P. Chen, and P. B. Wilson, IEEE Trans. Nucl. Sci. 32, 3524 (1985).

[48] P. Piot, C. Behrens, C. Gerth, M. Dohlus, F. Lemery, D. Mihalcea, P. Stoltz, and M. Vogt, Phys. Rev. Lett. 108, 034801 (2012). 
[49] M. Hüning, H. Schlarb, P. Schmüser, and M. Timm, Phys. Rev. Lett. 88, 074802 (2002).

[50] P. Emma, M. Venturini, K. L. F. Bane, G. Stupakov, H.-S. Kang, M. S. Chae, J. Hong, C.-K. Min, H. Yang,
T. Ha, W. W. Lee, C. D. Park, S. J. Park, and I. S. Ko, Phys. Rev. Lett. 112, 034801 (2014).

[51] G. Mishra and G. Sharma, Prog. Electromagn. Res. M 36, 47 (2014). 